\title{
Nanoparticle-induced negative differential resistance and memory effect in polymer bistable light-emitting device
}

\author{
Ricky J. Tseng, Jianyong Ouyang, Chih-Wei Chu, Jinsong Huang, and Yang Yang ${ }^{a)}$ \\ Department of Materials Science and Engineering, University of California-Los Angeles, Los Angeles, \\ California 90095
}

(Received 16 October 2005; accepted 2 February 2006; published online 21 March 2006)

\begin{abstract}
Recently, electrical bistability was demonstrated in polymer thin films incorporated with metal nanoparticles [J. Ouyang, C. W. Chu, C. R. Szmanda, L. P. Ma, and Y. Yang, Nat. Mater. 3, 918 (2004)]. In this letter, we show the evidence that electrons are the dominant charge carriers in these bistable devices. Direct integration of bistable polymer layer with a light-emitting polymer layer shows a unique light-emitting property modulated by the electrical bistability. A unique negative differential resistance induced by the charged gold nanoparticles is observed due to the charge trapping effect from the nanoparticles when interfaced with the light-emitting layer. (C) 2006 American Institute of Physics. [DOI: 10.1063/1.2187407]
\end{abstract}

Self-assembled nanoparticles have generated enormous interest in building electronic devices by utilizing their special physical and chemical properties through the nanostructures. ${ }^{1}$ Their enhanced optoelectronic properties have made semiconductor nanoparticles attractive for use in photovoltaic $^{2}$ and electroluminescence ${ }^{3}$ devices. Moreover, metal nanoparticles have sparked intense research on various device applications, such as rectifiers, ${ }^{4}$ transistors, ${ }^{5}$ and chemical $^{6}$ and biological sensors. ${ }^{7}$ Among the nanoparticles, the organic molecules capped metal nanoparticles, synthesized by two phase arrested growth ${ }^{8}$ and stabilized by alkanethiol with different chain lengths, ${ }^{9}$ are particularly attractive when combined with polymers due to their enhanced solubility.

Recently, an electronic memory device has been realized by this class of polymer-nanoparticle composites. ${ }^{10}$ These memory devices usually utilize hybrid composites or organic/nanoparticles/organic layered structures. ${ }^{11-13}$ The operation principle is based on electrical conductance switching, which leads to bistable states with an ON/OFF ratio larger than three orders of magnitude. One of the essential features is the nonlinear current-voltage characteristics [e.g., conductance switching and negative differential resistance (NDR)] which would allow further development of new information processing system. The NDR effect was recently reported by Tang et al. ${ }^{14}$ in molecular and organic electronic devices. These memory devices are worth more attention as well as further investigation to achieve practical applications. In this letter, we investigate the dominant carrier property in polymer bistable devices in order to understand the role of gold nanoparticles in device operation. We also report a polymer bistable light-emitting device (LED) based on direct integration of an electrically bistable polymer (EBP) composite with a light-emitting polymer (LEP). Finally, the unique light-emitting property modulated by the bistable effect, and NDR induced by the charge trapping in nanoparticles is studied. These integrated devices may provide practical applications in organic electronics using nanomaterials, such as memories, multifunctional devices, and electronic recordable papers.

\footnotetext{
${ }^{a)}$ Electronic mail: yangy@ucla.edu
}

Figure 1 shows the typical device structures of (a) polymer bistable device, and (b) polymer bistable light-emitting device. The EBP layer of polymer bistable device consists of a blend of $1.2 \mathrm{wt} \%$ polystyrene (PS), $0.4 \mathrm{wt} \%$ 8-hyroxyquinoline (8HQ), and $0.4 \mathrm{wt} \%$ dodecanethiol capped gold nanoparticles (AuNP). The polymer bistable LED structure can be presented as indium tin oxide (ITO)/ PEDOT $(40 \mathrm{~nm}) / \mathrm{LEP} \quad(100 \mathrm{~nm}) / \mathrm{EBP} \quad(30 \mathrm{~nm}) / \mathrm{Cs}_{2} \mathrm{CO}_{3}$ (few nanometers)/Al $(60 \mathrm{~nm})$ [PEDOT stands for poly $(3,4-$ ethylenedioxythiophene): polystyrenesulfonate.)]. The LEP is polyfluorene co-polymer (5BTF8), consisting of $95 \mathrm{wt} \%$ poly (9,9-dioctylfluorene) and $5 \mathrm{wt} \%$ poly (9,9-dioctylfluorene-co-benzothiadiazole). ${ }^{15}$ Cesium carbonate is used as the electron injection layer. ${ }^{16}$ The chemical structures of the organic molecules and the polymers used are also shown in Fig. 1. Current-voltage $(I-V)$ characteristics of devices were measured by an HP 4155 semiconductor parameter analyzer. In forward scan, the ITO electrode (anode) was positively biased. The electroluminescence was measured by a Photoresearch PR650 spectrophotometer. The transmission electron microscopy was imaged by JEOL JEM-2000FX, with

(a)

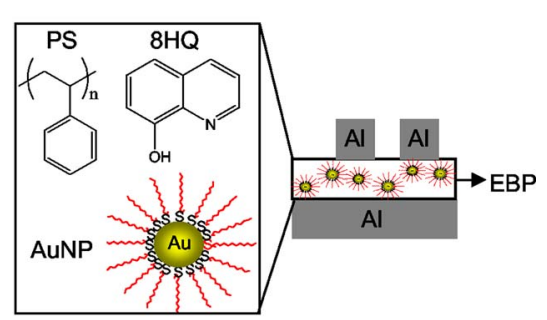

(b)

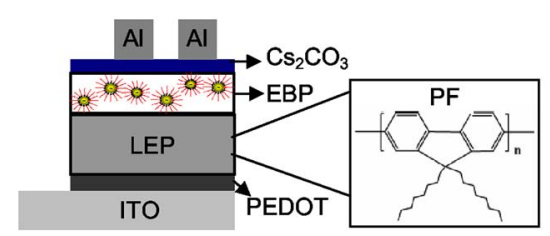

FIG. 1. (Color online) The device structures of (a) polymer bistable device, and (b) polymer bistable LED. The polymer-nanoparticle composite (PS $+8 \mathrm{HQ}+\mathrm{AuNP})$ is used as the EBP. Chemical structures of the organic materials used are also shown. 
(a)

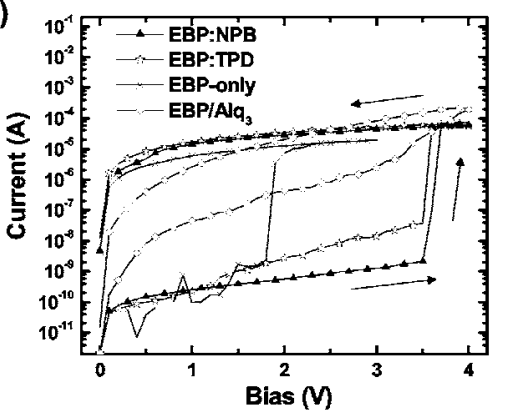

(b)

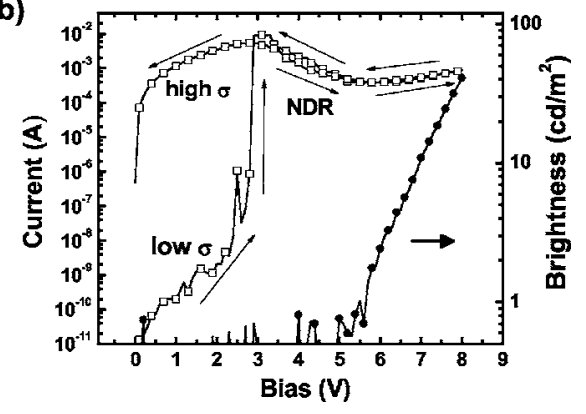

FIG. 2. (a) $I-V$ characteristics of polymer bistable devices. NPB and TPD with 0.08 wt $\%$ concentration are blended into the EBP films or an extra layer of $\mathrm{Alq}_{3}$ is inserted for carrier type study. (b) $I$ - $V$-luminescence characteristics of polymer bistable LED ( $\sigma$ represents the conductivity).

samples prepared by drop casting the gold nanoparticle solution on copper grids.

Although the operation mechanism has been reported for the polymer bistable device, ${ }^{10}$ further investigation of the carrier type (either hole or electron) in the conductance switching is still not fully realized. In order to understand the carrier type, four types of EBP films were prepared: (i) The original EBP film (termed as EBP only), (ii) the EBP films blended with two different hole-transporting materials, 4, 4' -bis-(1-naphthyl-N-phenylamino)-biphenyl (NPB) and $\mathrm{N}, \mathrm{N}^{\prime}$-diphenyl-N, $\mathrm{N}^{\prime}$-bis(3-methylphenyl)-(1, 1'-biphenyl)$4,4^{\prime}$-diamine (TPD), and (iii) the EBP layer combined with electron transporting layer tris-(8-hydroxyquinoline) aluminum $\left(\mathrm{Alq}_{3}\right)$. It is known that the electron mobility in holetransporting materials is usually low, and they are considered as electron blockers. ${ }^{17} \mathrm{NPB}$ and TPD, with a concentration of $0.08 \mathrm{wt} \%$ and $\mathrm{Alq}_{3}$, as a separate layer of $5 \mathrm{~nm}$ thickness were used. (We term these films: EBP:NPB film, EBP:TPD film, and EBP/ $/ \mathrm{Alq}_{3}$.) The $I-V$ curves of devices using these four films are shown in Fig. 2(a). The current starts from a low current level, about $1 \times 10^{-10} \mathrm{~A}$ in the low voltage range $(<2 \mathrm{~V})$, and abruptly increases to $1 \times 10^{-10} \mathrm{~A}$ at a turn-on bias. The two conductivity states differ by five orders of magnitude. The turn-on bias for EBP only, EBP:NPB, and EBP:TPD devices are $1.8 \mathrm{~V}, 3.5 \mathrm{~V}$, and $3.5 \mathrm{~V}$, respectively. However, for the $\mathrm{Al} / \mathrm{EBP} / \mathrm{Alq}_{3} / \mathrm{Al}$ device, we observe slight hysteresis and no obvious switching behavior.

The switching mechanism of the memory device is attributed to the electric-field induced charge (electron) transfer from 8HQ to AuNP. ${ }^{10}$ After the charge transfer, 8HQ become partially positively charged and AuNP become negatively charged. Hence, in the high conductivity state the tunneling process takes place through either the 8HQ or AuNP. In the case of EBP:NPB and EBP:TPD, the higher turn-on bias indicates that the electron transfer from 8HQ to AuNP becomes more difficult due to the potential barrier created
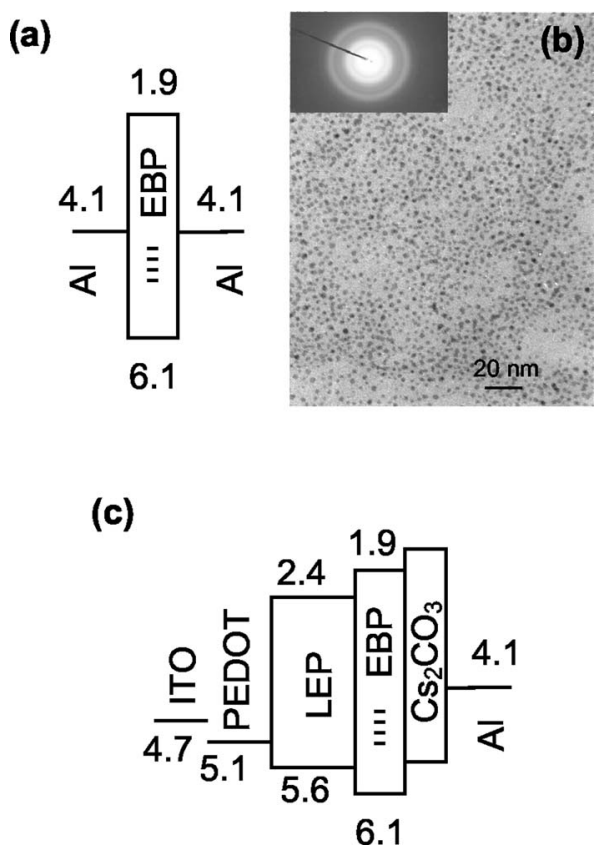

FIG. 3. (a) Schematic energy diagram of polymer bistable device, (b) TEM image of dodecanethiol capped gold nanoparticles with size distribution from 1.6 to $4.4 \mathrm{~nm}$, and, (c) schematic energy diagram of polymer bistable LED with the HOMO and LUMO levels of materials used and trap levels of dodecanethiol capped gold nanoparticles (short lines), in electron volts and with respect to vacuum level.

from the electron blocker materials. On the other hand, for the $\mathrm{EBP} / \mathrm{Alq}_{3}$ device, the switching phenomenon was smeared out by the $\mathrm{Alq}_{3}$ layer due to its better electron transport property. After the charge transfer, electrons may tunnel through the AuNPs in the high conductivity state; therefore, the currents are approximately the same due to the relatively similar AuNP concentration. In comparison, we did not observe any significant change by adding the hole blocking material; suggesting that the holes are minority carriers in the transport mechanism. Additionally, in the polymer bistable LEDs, the electron transport through the EBP layer results in light emission, which will be discussed in detail in the later part of this letter. From these observations, we conclude that the electrons are the majority carriers in the electrical polymer bistable device.

The polymer bistable LED with combined EBP and LEP layers is demonstrated in Fig. 2(b). The measured $I$ - $V$ curves for the device follow the arrow signs in forward and reverse bias scans ( 0 to 8 to $0 \mathrm{~V})$. An abrupt jump in current by five orders of magnitude is observed at $2.6 \mathrm{~V}$. After turning to the high conductivity state, the current then decreases from 3 to $5.6 \mathrm{~V}$, accompanying a peak-to-valley ratio of 20 . It shows an N-shaped peak, which defines a NDR.

The schematic energy diagrams, corresponding to the polymer bistable device and polymer bistable LED are shown in Figs. 3(a) and 3(c), respectively. These energy levels show the highest occupied molecular orbital (HOMO) level and the lowest unoccupied molecular orbital (LUMO) level for different materials used. The short lines in the EBP layer represent the trap ${ }^{14}$ levels of gold nanoparticles due to the nonconducting chain of the capped dodecanethiol molecule and the nanoparticles can be charged. ${ }^{18}$ From Figs. 2(a) and 3(a), single EBP layer device shows no NDR, since there is no potential barrier between the electrode and AuNP, and the conduction mechanism is through tunneling. 
In the polymer bistable LED device, [Figs. 2(b) and $3(\mathrm{c})$ ], from 3 to $5.6 \mathrm{~V}$, the electrons injected from the cathode are not able to pass through the EBP layer, since the trap level is not matched with the LUMO of the LEP. This corresponds to the current decrease with increasing voltage, or the NDR behavior. The NDR suggests that the nanoparticles behave as charge traps in this unique structure where the electron tunneling is prohibited. Since there is a size dispersion of the gold nanoparticles in the transmission electron microscope (TEM) image, as shown in Fig. 3(b) (size distribution range from 1.6 to $4.4 \mathrm{~nm}$ ), the energy level of AuNP is not a fixed value. These electrons might first fill the deeper empty states, and subsequently fill the shallower levels. ${ }^{24}$ When the bias is larger than 5.6 V [Fig. 3(c)], corresponding to the shallower trap level close to the LUMO of LEP, electrons injected from the cathode can propagate to the LEP layer, and electroluminescence is observed. On the contrary, when the bias is smaller than $5.6 \mathrm{~V}$, the filled trap state lies below the LUMO of LEP, and this is basically a single carrier device with no electroluminescence. Additionally, the NDR regions overlap nicely while biasing upward and downward as shown in Fig. 3(c). This suggests that the repeated observation of NDR can be reliably regarded as an evidence of charge-trapping levels of nanoparticles, possibly related to the quantized energy of nanoparticles. It is worth mentioning that only polymer bistable LED devices having AuNPs in the EBP layer show this kind of NDR phenomenon. Finally, the voltages across the whole device during light emission must include the following: The turn-on bias of EBP layer, hole and electron injection bias from the electrodes, and the barrier between the trap level of AuNP and LUMO of LEP. Although the exact trapping level of AuNP is still not clear, the charge trapping effect in this device is evident. This also shows electrons as the dominant carriers in EBP layer in the polymer bistable LED, consistent with the observed majority carrier of electrons in polymer bistable device.

Organic electronic devices with NDR behavior are rarely reported. The NDR behavior was recently reported in organic bistable devices with metal nanoparticles ${ }^{13,14}$ as well as in polyaniline nanofibers with gold nanoparticles. ${ }^{19}$ This provides very exciting insight into nanomaterial science and device physics for future information processing systems by using NDR. ${ }^{20,21}$ The NDR is usually observed in resonant tunneling semiconductor devices ${ }^{22}$ and nanocrystal embedded metal-oxide-semiconductor devices, ${ }^{23}$ attributed to the quantum confinement effect. In our case, the NDR is associated with the trapped charges in the nanoparticles, similar to the semiconductor devices with nanocrystals. ${ }^{24}$ The resonant tunneling behavior is excluded from consideration due to the relatively large thickness of the LEP layer.

In summary, we show that the electrons are the majority carriers in polymer bistable devices. A solution-processed polymer electrical bistable LED is also demonstrated. The unique polymer-nanoparticle composite integrated with LEP serves as a unique material system to fabricate functional devices. The device shows dual functions of memory effect and light-emitting behavior. The NDR, nicely overlapped in both scanning directions, is induced by the charge trapping effect of the gold nanoparticles. The NDR is attributed to the injected electrons filling the trap states of the nanoparticles. This is further confirmed by the electroluminescence in LEP after the electrons overcome the barrier from the trap level to the LUMO of LEP. This observation suggests that the nanoparticles behave as charge traps only when they are in a unique structure where the tunneling is prohibited. The NDR phenomenon disappeared when the two films are not in a close contact, such as in a single EBP layer device. By combining nanoparticles and organic materials, these devices pave a way for nanotechnology and future organic electronics.

This work was supported by the Microelectronics Advanced Research Corp. (MARCO) Focus Center on Functional Engineered Nano Architectonics (FENA) at University of California, Los Angeles and the Air Force Office of Scientific Research.

${ }^{1}$ S. Hoeppener, R. Maoz, S. R. Cohen, L. F. Chi, H. Fuchs, and J. Sagiv, Adv. Mater. (Weinheim, Ger.) 14, 1036 (2004).

${ }^{2}$ W. U. Huynh, J. J. Dittmer, and A. P. Alivisatos, Science 295, 2425 (2002).

${ }^{3}$ S. A. Carter, J. C. Scott, and P. J. Brock, Appl. Phys. Lett. 71, 1145 (1997).

${ }^{4}$ T. Reda, A. F. Collings, C. Barton, and P. Lukins, J. Phys. Chem. B 107, 13774 (2003).

${ }^{5}$ K. I. Bolotin, F. Kuemmeth, A. N. Pasupathy, and D. C. Ralph, Appl. Phys. Lett. 84, 3154 (2004).

${ }^{6}$ S. M. Briglin, T. Gao, and N. S. Lewis, Langmuir 20, 299 (2004).

${ }^{7}$ R. Elghanian, J. J. Storhoff, R. C. Mucic, R. L. Letsinger, and C. A. Mirkin, Science 277, 1078 (1997).

${ }^{8}$ M. Brust, M. Walker, D. Bethell, D. J. Schiffrin, and R. J. Whyman, J. Chem. Soc., Chem. Commun. 1994, 801 (1994).

${ }^{9}$ Y. Joseph, I. Besnard, M. Rosenberger, B. Guse, H. G. Nothofer, J. M. Wessels, U. Wild, A. Knop-Gericke, D. Su, R. Schlögl, A. Yasuda, and T. Vossmeyer, J. Phys. Chem. B 107, 7406 (2003).

${ }^{10}$ J. Ouyang, C. W. Chu, C. R. Szmanda, L. P. Ma, and Y. Yang, Nat. Mater. 3, 918 (2004).

${ }^{11}$ L. P. Ma, J. Liu, and Y. Yang, Appl. Phys. Lett. 80, 2997 (2002).

${ }^{12}$ L. P. Ma, S. Pyo, J. Ouyang, Q. F. Xu, and Y. Yang, Appl. Phys. Lett. 82, 1419 (2003).

${ }^{13}$ L. D. Bozano, B. W. Kean, V. R. Deline, J. R. Salem, and J. C. Scott, Appl. Phys. Lett. 84, 607 (2004).

${ }^{14}$ W. Tang, H. Shi, G. Xu, B. S. Ong, Z. D. Popovic, J. Deng, J. Zhao, and G. Rao, Adv. Mater. (Weinheim, Ger.) 17, 2307 (2005).

${ }^{15}$ C. I. Wilkinson, D. G. Lidzey, L. C. Palilis, R. B. Fletcher, S. J. Martin, X. H. Wang, and D. D. C. Bradley, Appl. Phys. Lett. 79, 171 (2001).

${ }^{16}$ T. Hasegawa, S. Miura, T. Moriyama, T. Kimura, I. Takaya, Y. Osato, and H. Mizutani, Proceedings of the 2004 Society for Information Display (SID) International Symposium, Digest of Technical Papers, p. 154.

${ }^{17}$ S. T. Zhang, Z. J. Wang, J. M. Zhao, Y. Q. Zhan, Y. Wu, Y. C. Zhou, X. M. Ding, and X. Y. Hou, Appl. Phys. Lett. 84, 2916 (2004).

${ }^{18}$ S. Chen, R. S. Ingram, M. J. Hostetler, J. J. Pietron, R. W. Murray, T. G. Schaaff, J. T. Khoury, M. M. Alvarez, and R. L. Whetten, Science 280, 2098 (1998).

${ }^{19}$ R. J. Tseng, J. Huang, J. Ouyang, R. B. Kaner, and Y. Yang, Nano Lett. 5, 1077 (2005).

${ }^{20}$ J. D. Le, Y. He, T. R. Hoye, C. C. Mead, and R. A. Kiehl, Appl. Phys. Lett. 83, 5518 (2003).

${ }^{21}$ W. J. Yoon, S. Y. Chung, P. R. Berger, and S. M. Asar, Appl. Phys. Lett. 87, 203506 (2005).

${ }^{22}$ N. C. Kluksdahl, A. M. Kriman, D. K. Ferry, and C. Ringhofer, Phys. Rev. B 39, 7720 (1989).

${ }^{23}$ V. Ioannou-Sougleridis and A. G. Nassiopoulou, J. Appl. Phys. 94, 4084 (2003).

${ }^{24}$ Y. Shi, K. Saito, H. Ishikuro, and T. Hiramoto, J. Appl. Phys. 84, 2358 (1998). 